\title{
Unusual localization of an hydatid cyst: first reported case in Buenos Aires, Argentina
}

\section{ABSTRACT}

Hydatidosis is a parasitic infection caused by the tapeworm larva of Echinococcus spp. Its relevance lies in its wide distribution, great number of clinical cases and outstanding morbility. Hydatid infection of the orbit comprises far less than $1 \%$ of the total incidence. This is a case of a patient from Argentina complaining of a two-week evolution proptosis of the right eye. A microscopic examination revealed the presence of protoscolices of Echinococcus spp. in the fluid obtained during the surgical proceedings. The patient was treated with oral albendazole. To our knowledge, this is the first case of occular hydatidosis diagnosed in the city of Buenos Aires, Argentina.

Keywords: echinococcosis; orbit; Argentina.

[Braz J Infect Dis 2011;15(1):81-82] @Elsevier Editora Ltda.

Hydatidosis is a parasitic infection caused by the tapeworm larva of Echinococcus spp. In humans, $80 \%$ to $95 \%$ of hydatids develop in the liver and lungs. The orbit is one of the rarest sites to be involved and comprises far less than $1 \%$ of the total incidence. ${ }^{1}$ Until 1983 , about 15 cases of orbital cysts had been reported in In$\mathrm{dia}^{2}$ and 11 cases had been reported in South Africa by $1977 .{ }^{3}$ Recently, in a review between 1984 and 2006, 10 cases of orbital hydatid cyst were reported. ${ }^{4}$ In the Hospital de Clínicas, Buenos Aires, we found a case of hydatid cyst of the orbit in a patient from Neuquén, Argentina. The patient was a 41-year-old woman who lived in the countryside and worked in the manufacture of "chacinados", a kind of cured pork sausage. She complained of a two-week evolution proptosis of the right eye. A computed tomography (CT) scan revealed the presence of a well-delineated, ovoid, heterogeneous, tumorlike mass of $16 \times 24 \mathrm{~mm}$ in the right eye, confined to the orbit. A whitish cystic mass of the orbit was removed by surgical excision under general anesthesia. Rupture occurred with loss of an uncolored fluid and the microscopic examination revealed the presence of protoscolices of Echinococcus spp (Figure 1). After surgical excision, a subsequent lavage of the area

Figure 1: Protoscolex of Echinococcus spp.

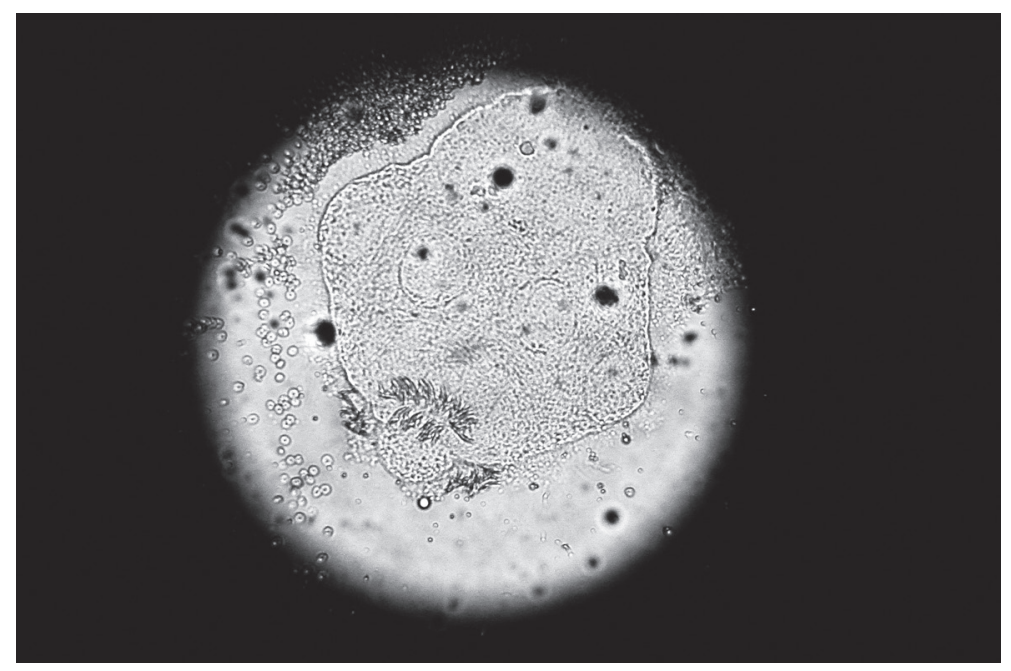

Authors

Claudia Irene Menghi ${ }^{1}$ Claudia Liliana Gatta ${ }^{2}$

${ }^{1}$ Biochemist Parasitologist, PhD - Head of Parasitology Laboratory, Hospital de Clinicas, University of Buenos Aires

${ }^{2}$ Biochemist Parasitologist - Research Assistant, Parasitology Division, Hospital de Clinicas, University of Buenos Aires

Submitted on: 04/30/2010 Approved on: 05/02/2010

Correspondence to: Dra. Claudia Menghi Parasitology Division, Hospital de Clínicas, Faculty of Pharmacy and Biochemistry, University of Buenos Aires

Teodoro García 23507 A 1426 Buenos Aires, Argentina cmenghi@fibertel.com.ar

We declare no conflict of interest. 
with hypertonic saline solution and sodium bicarbonate was performed. The patient was started on oral albendazole in doses of $400 \mathrm{mg}$ twice a day, and received this drug in three cycles of 28 days, with intervals of 14 days without treatment.

Typically, occular hydatid cyst is unilateral and occurs with or without hydatid cysts located elsewhere in the body. ${ }^{5}$ Occular hydatid cysts should be considered in the differential diagnosis of unilateral proptosis, especially in endemic areas. The most frequent clinical findings are exophthalmos, chemosis, lid edema, visual impairment, and restriction of extraocular motility. ${ }^{5}$ Development of effective control programs should be intensified to control hydatidosis. To our knowledge, this is the first case of occular hydatidosis diagnosed in the city of Buenos Aires, Argentina.

\section{REFERENCES}

1. Turgut AT, Turgut M, Koşar U. Hydatidosis of the orbit in Turkey: results from review of the literature 1963-2001. Int Ophthalmol. 2004; 25:193-200.

2. Lamba PA, Bhatia PC, Jain M, Baweja U. Hydatid cyst of orbit. Indian J Ophthalmol. 1983; 31:23-5.

3. Sevel D, Sapeika RJ. Hydatid cyst of the orbit. Surv of Ophthalmol. 1977; 22:101-5.

4. Benazzou S, Arkha Y, Derraz S et al. Orbital hydatid cyst: review of 10 cases. J Craniomaxillofac Surg (2009). Pubmed DOI: $10.1016 /$ j.jcms.2009.10.001.

5. Selçuklu A, Öztürk M, Külahli I, Dogan H. Successful surgical management of an intraorbital hydatid cyst through a transmaxillary approach: case report. Skull Base 2003; $13: 101-5$ 